\title{
Philosophiques
}

\section{La raison comme lemme philosophique ou « Der Instinkt der Vernünftigkeit » - pour accoster Hegel}

\section{Jean-Luc Gouin}

Volume 23, numéro 2, automne 1996

URI : https://id.erudit.org/iderudit/027397ar

DOI : https://doi.org/10.7202/027397ar

Aller au sommaire du numéro

Éditeur(s)

Société de philosophie du Québec

ISSN

0316-2923 (imprimé)

1492-1391 (numérique)

Découvrir la revue

Citer cet article

Gouin, J.-L. (1996). La raison comme lemme philosophique ou « Der Instinkt der Vernünftigkeit " - pour accoster Hegel. Philosophiques, 23(2), 285-303.

https://doi.org/10.7202/027397ar
Résumé de l'article

Bien que l'on associat traditionnellement pensée hégélienne et affirmation péremptoire de la rationalité de la réalité (die Vernünftigkeit der Wirklichkeit), il n'est pas inopportun de tenter de saisir le fil d'Ariane qui, progressivement, conduit le philosophe de l'Idéalisme absolu à littéralement arraisonner la Raison. On comprendra dès lors que cette Raison est peut-être moins un FIAT originel né de l'arbitraire - fût-il celui, génial, d'un puissant esprit pensant qu'une simple CONSTANTE du réel : nous sommes de raison dans la Raison. Aussi, lutter contre celle-ci constitue sans doute non tant un acte de Liberté que d'entêtement Reste donc à s'en faire une alliée. Il nous faut épouser Athéna - et cultiver die Rose im Kreuze der Gegenwart, la rose dans la croix du présent. 


\title{
LA RAISON COMME LEMME PHIILSOPHIQUE OU « DER INSTINKT DER VERNÜNFIIGKEIT » - POUR ACCOS IER HEGEl -
}

\author{
PAR \\ JeAN-LUC GOUIN
}

Die Gedanken nicht bloß unsere Gedanken, sondern zugleich das Ansich der Dinge.

\begin{abstract}
RÉSUMÉ : Bien que l'on associät traditionnellement pensée hégélienne et affirmation péremptoire de la rationalité de la réalité (die Vernünftigkeit der Wirklichkeit), il n'est pas inopportun de tenter de saisir le fil d'Ariane qui, progressivement, conduit le philosophe de l'Idéalisme absolu à littéralement arraisonner la Raison. On comprendra dès lors que cette Raison est peut-être moins un FIAT originel né de l'arbitraire fút-il celui, génial, d'un puissant esprit pensant-qu'une simple CONSTANTE du réel : nous sommes de raison dans la Raison. Aussi, lutter contre celle-ci constitue sans doute non tant un acte de Liberté que d'entêtement. Reste donc à s'en faire une alliée. Il nous faut épouser Athéna - et cultiver die Rose im Kreuze der Gegenwart, la rose dans la croix $d u$ présent.

ABSTRACT: We will analyze how Hegel leads us from Absolute Idealism to the well-known thesis: Reality is Rationality. We will see thus that Reason is not produced by an arbitrary and theoretical decision but, on the contrary, that Reason reveals itself as a constant of Reality. The struggle against Reason cannot appear, therefore, as an act of freedom but as a form of the stubbomness which characterizes the activity of Reason. In this process, Reason is its own ally.
\end{abstract}

Il s'agit pour le philosophe de " transformer l'évidence universelle de l'être du monde (pour lui la plus grande des énigmes) en quelque chose qui se comprenne".

Ces lignes ne sont pas de Hegel. Pourtant elles pourraient aisément se loger en son texte sans qu'il n'y paraisse. Cette éventualité revêt un sens d'autant plus lourd que Husserl, le penseur qui a énoncé cette idée ${ }^{1}$, connaissait à peine son non moins réputé

1. E. Husserl, La Crise des sciences européennes et la Phénoménologie transcendantale. Paris, Gallimard, NRF, 1976 [1936/1954], § 53, p. 205. Idée qui renvoie d'ailleurs à une qualité fondamentale bien connue des 
compatriote d'un siècle son aîn $\dot{e}^{2}$. Au fond, il n'y a peut-être là rien qui doive... étonner si, comme le prétend une commentatrice de notre auteur, "le propre de la philosophie est d'aborder ce qui est en sa totalité en recherchant d'un même mouvement ce qui fait que ce qui est est tel ${ }^{3}$."

Bien sûr, il n'est point aisé de créer le consensus autour du concept même de philosophie. Il semble toutefois qu'il y ait en génėral accord sur deux dimensions fondamentales, à savoir : qu'elle consiste en un questionnement qui cherche la vérité mais encore, et plus spécifiquement, la vèritè de la totalité. C'est dire dès l'abord son caractère radical : la philosophie aspire à dépasser l'opinion et, d'autre part, à ne pas réduire sa réflexion à des domaines repliés sur eux-mêmes, ètanches à tout autre. Elle cherche de la sorte à éviter le ghetto des savoirs qui ne mettent pas ces différents plans en relation, entre eux et/ou avec la totalité.

L'objet de la philosophie, c'est donc nulle autre chose que le monde bien réel dont on cherche le sens. De fait, le philosophe pose à celui-ci la laconique mais combien troublante question : "Que faistu là, monde? "Ce que notre penseur exprime avec simplicité dans une formule fort humble, laquelle ne cache point pour autant ses ambitions : "Saisir et comprendre ce qui est, telle est la tâche de la philosophie ${ }^{4}$."

Le sens et la totalité constituent les deux pôles du penser philosophique. Telles les abscisse et ordonnée du graphe à la conquéte de l'espace, il est essentiel de considérer le projet hégélien dans toute lamplitude de ce balayage à la fois horizontal et vertical du réel. Aussi, l'avertissement suivant se voit-il justifié :

La fidèlité à ce qui fait l'essentiel de l'entreprise hégélienne impose de la sorte une prise en compte première des perspectives les plus vastes (celles oủ la totalité se donne à connaitre comme totalité) avant que l'on vienne à braquer l'objectif sur telle partie du tout ${ }^{5}$.

Hegel insista longuement sur cette dimension de totalité - qu'il nous faut ne jamais perdre de vue, sans quoi notre regard et notre compréhension du texte mutilent véritablement sa pensée : "Tout ce

enfants comme des philosophes authentiques, Aristote en particulier : Le commencement de toutes les sciences, c'est l'étonnement de ce que les choses sont ce qu'elles sont. "Métaphysique, Alpha, 2. Le "Die Instinkt der Vernünftigkeit "du titre signifie : "L'instinct de la rationalité ", que Gibelin préféra traduire par: instinct rationnel. Philo., p. 232 (203); et la citation en exergue pose que "Les pensées ne sont pas simplement nos pensées, mais en même temps l'en-soi des choses. "Enc., \$ 41 , add. $n^{\circ} 2$, p. 499 (VIII, 116) [voir le code des sigles et des références en toute fin de texte].

2. Voir la note complémentaire en fin de texte.

3. Gwendoline Jarczyk, Système et liberté dans la Logique de Hegel Paris, Aubier-Montaigne, 1980 , p. 21

4. "Das was ist zu begreifen, ist die Aufgabe der Philosophie. "Droit, p. 57 (VII, 26). * Dans la connaissance, il s'agit d'une façon générale d'ôter son caractère étranger au monde objectif qui nous fait face et. comme on a coutume de dire, de nous retrouver en lui, ce qui signifie la mème chose /que ramener l'être objectif au concept, qui est notre Soi le plus intime (als das Objektive auf den Begriff zurückzuführen, welcher unser innerstes Selbst ist). Enc., § 194, add. $\mathrm{n}^{\circ}$ 1, p. 608 (VIII, 351). Cf. aussi Enc.-3, § 447, add., p. 546 et Droit, $\$ 4$, add., p. 72 .

5. G. Jarczyk et P.-J. Labarrière, dans leur Présentation à Log.-1, p. XX-XXI. 
qui mérite le nom de philosophie, dira-t-il, a toujours eu à son fondement/la conscience d'une unité absolue ${ }^{6}$."

Par ailleurs, si la philosophie vise le comprendre et cherche le sens, que s'ingénie-t-elle à appréhender sinon la vérité de la réalité - la signification des choses ? Or s'il y a quelque chose à comprendre et que, par là, il est impliqué qu'il y ait de la vérité et du sens, nous optons de suite pour la rationalité.

Déjà le " bon sens" dont chacun s'estime bien pourvu, comme le faisait remarquer Descartes, se fonde sur cette évidence de la rationalité - c'est-à-dire la présence d'un cœfficient d'intelligibilité transitant les choses. L'univers des sciences, pour sa part, la reçoit avec empressement sous ses lois et ses théories. Et les réfutations de celles-ci au long de l'histoire du savoir témoignent non pas de la faillite de la raison, mais bien sûr de son dépassement en des champs toujours plus vastes. Reste maintenant l' au-delà " de cet espace. Car si de fait il est généralement admis que la raison " règle " la nature au point où "nous y sommes accoutumés et nous n'en faisons pas grand $\operatorname{cas}^{7}$ ", il nous faut ou bien poser l'arrêt, la limite de la raison, ou bien identifier sa présence par delà les frontières plus ou moins justifiées ètablies jusqu'à maintenant. La raison, estime Hegel dans une envolée aux intonations augustiniennes (et son œuvre s'en veut la démonstration continue), est partout ou nulle part. Or si déjà un coin du réel ne lui échappe point, le défi de la totalité rationnelle s'en trouve par là mème lancé ${ }^{\text {. }}$.

Car si les mots "comprendre ", "connaître " et "vérité " ont un sens, "comprendre ce qui est "implique que ce qui est soit en quelque façon compréhensible, c'est-à-dire rationnel. On saisira le message exprimé par le professeur dès sa leçon inaugurale à Berlin : "Le courage de la vérité, la foi en la puissance de l'esprit, sont la première condition de l'étude philosophique ${ }^{9}$."

Mais ne devons-nous pas constater ici l'existence d'un postulat qui, par sa "brutalité " de fait premier, se voit susceptible d'être réfuté ou pour le moins interrogé ? Hegel ne dénature-t-il pas la raison elle-mēme en la fondant somme toute sur un acte de foi, lorsqu'il maintient que "la seule idée qu'apporte la philosophie est la simple idée de la Raison-l'idée que la Raison gouverne le monde ${ }^{10} "$ ?

6. "Das Bewußtsein einer absoluten Einheit. "Enc., § 213, add., p. 616 (VIII, 369).

7. "Wir sind dergleichen gewohnt und machen nicht viel daraus. "Raison, p. 56 (37). Cf. Philo., p. 144-145 et 199, ainsi que Droit. Préface, passim

8. "De même le germe porte en lui la nature entière de l'arbre ainsi que le goūt et la forme des fruits, de même les premières traces de l'Esprit contiennent virtuellement toute l'histoire (Und wie der Keim die ganze Natur des Baumes, den Geschmack, die Form der Früchte in sich trägt, so enthalten auch schon die ersten Spuren des Geistes virtualiter die ganze Geschichte), " Raison, p. 83 (61).

9. Bernard Bourgeois incorpora à sa traduction de la Logique de l'Encyclopédie cette allocution, d'ailleurs fort bien ramassée, du 22 octobre 1818 ; Enc., p. 148. On lira également "que la forme de la pensée est la forme absolue (Daß die Form des Denkens die absolute ist) et que la vérité apparaît en elle comme elle est en et pour soi, c'est là l'affirmation de la Philosophie en général. "Enc., \$ 24, add. n 3, p. 480 (VIII, 87). Aussi, cf. Philo., p. 112.

10. "Der einzige Gedanke, den sie mitbringt, ist aber der einfache Gedanke der Vernunft, daß die Vermunft die Welt beherrscht. "Raison p. 47 (28). Formule 
On se doute qu'une telle affirmation ne fit pas l'unanimitè au sein des milieux intellectuels et philosophiques. Des pans entiers du réel, oppose-t-on à l'auteur, ne peuvent ètre considèrés absolument rationnels. La réalité, de façon générale, n'est-elle pas tout au contraire un jeu confus, un va-et-vient invraisemblable où le mal, la souffrance, l'exploitation de l'homme, la bêtise et la guerre possèdent toujours les meilleures mains?

D'autre part, dans la lignée de la tradition idéaliste, on a tôt fait de rappeler à Hegel que la sphère de la raison reste distincte de la réalité " sensible". La scissure entérinée par Platon entre le monde des Idées et celui de ses ombres se poursuit chez Descartes (jusquelà essentiellement vêhiculée dans sa forme christianisée), qui la radicalise en la fondant sur l'ego cogito d'où émane le dualisme bien connu de la pensée et de l'étendue.

Kant se penche de façon systematique sur cette opposition en tentant de rapprocher les deux univers par les catégories de la raison et les formes de la sensibilité. Il reste toutefois fidèle à la tradition en maintenant que la rationalité dans le monde est toujours celle que l'homme y a mise lui-même. Conséquemment, ce monde demeure pour ainsi dire viscéralement chaotique.

Face à cette attitude, notre philosophe considère que la raison demeure en réalité l'unique juge : l'histoire est-elle rationnelle, la réalité sensible de façon générale est-elle rationnelle? Gui peut dire sinon la réflexion, la pensée, le travail de la raison avec son objet et ses questions ${ }^{11}$ ? On ne saurait en effet poser a priori de jugement sur quelque objet sans l'avoir préalablement réfléchi, sans l'avoir littéralement interrogé : le vrai et le faux sont toujours les enfants d'une réflexion.

Dès lors, ou bien le jugement découle lui-mème d'un mouvement irrationnel exhalé depuis un sentiment ou une conviction - et c'est alors une forme de pari qui ne peut ètre qualifié de "véridique "(tout au plus peut-il s'estimer hypothétique, assertorique au mieux et en tous les cas problématique). Ou bien il est l'œuvre du travail de la raison par les ressources de laquelle ce jugement parvient au vrai. Or si par un examen de cette sorte on est amenè à considérer une "tranche "de la réalité comme ètant en l'occurrence non rationnelle (ou a-rationnelle), cette a-rationalité resterait tout de mème tributaire de la raison qui l'énonce.

Il apparaît donc que l'a-rationalité ne revèt un sens que dans un discours de raison sans lequel nous aboutirions à une "a-rationalité irrationnelle", parce que susceptible d'acquiescement ou de rejet selon des critères eux-mèmes insaisissables sinon farfelus -

qui rèsume à elle seule tout le projet philosophique de notre auteur, et qui va à la rencontre de cette autre non moins fracassante : "Ce qui est rationnel est effectif, et ce qui est effectif est rationnel (Was vemünftig ist das ist wirklich; und was wirklich ist das ist vemünftig). "Droit, p. 55 (VII, 24). Effectivité (réalitê effective ou véritable : Wirklichkeit) est ici à distinguer notamment de Existenz, Realität (réalitê), Dasein (être-là), Sein (ètre) ou Wesen (essence, ou parfois : être), a fortiori de Schein (apparence) ou de Erscheinung (phènomène).

11. D'où la consêquence, en parallèle aux canons de la note précédente : " Ce qui n'est pas rationnel n'a aucune vérité, ou ce qui n'est pas conceptuellement conçu n'est pas (Was nicht vernünftig ist, hat keine Wahrheit, oder was nicht begriffen ist, ist nicht). "Ph-2, p. 100 (III, 404). 
nommément la simple certitude ou prétention de dire vrai sans qu'il soit possible d'interroger la validité du propos. Procéder à une certaine investigation de la réalité de façon à identifier s'il y a, ici ou là, effectivement ou non " rationalité ", revient donc constamment à buter contre un faux problème, à savoir : la raison peut-elle ètre autre que rationnelle?

Par ailleurs et plus spécifiquement, maintenir l'opposition entre le monde sensible et celui de la raison conduit au même titre à poser la question de la source de cette opposition. À cet égard, et malgré tout ce qui les distingue, Husserl rejoint une fois encore son illustre prédécesseur. Ils poseraient certainement de concert cette même question : "Est-ce que la raison et l'ètant doivent ètre séparés, alors que la raison connaissante détermine ce qui est étant ${ }^{12}$ ? "

La thèse de la " raison pure ", c'est-à-dire se suffisant à elle-même (face au sensible), se voit donc marquée elle aussi du fer rouge de la réflexion qui l'a fait " naitre ". Car en effet, ou cette raison présumée pure se dévoile à notre connaissance de cette façon (par raisonnement), ou bien elle est simplement postulée. Or postuler une théorie ne constitue rien de plus qu'un vouloir être, une assertion susceptible d'être révoquée par une autre dont l'auteur maintiendrait également le bien-fondé.

Bref, affirmer l'opposition pensée-réalité sensible s'appuie au fond sur une simple opinion. Et si d'aventure ce rapport d'opposition devait s'avérer réel, authentique, il ne saurait l'être qu'au nom de la raison qui en foumirait du coup l'argumentation. À la limite, la raison se prouverait elle-même en prouvant son contraire. Elle revient donc à son rôle constitutif et à sa position initiale, à savoir : source de tout jugement de sens sur le monde.

Considérer que la raison reste en retrait, isolée du monde, est une position qui ne résiste pas à l'analyse. Elle renvoie au foyer de sens qui (pré)autoriserait sa propre valeur : la raison supposément retranchée du monde sensible provient, émane, de la raison qui l'a pensée. Elle n'apparait donc nullement originaire ou indépendante, et la thèse se voit ainsi déboutée. Une raison autosuffisante face à un monde qui en serait pour ainsi dire la matière aveugle, selon le mot de Kant $^{13}$, nous projette vers une raison plus englobante dans laquelle ce monde "matériel "cesse d'incarner le spectre de l'irréductible.

La " scission " est tout au plus un instrument utilitaire pour une gnoséologie locale, laquelle s'embrouille aussitōt lorsqu'elle prétend à l'universalité ${ }^{14}$. En toute cohérence, et en dernière analyse, ce que nous appellerions le dichotomisme ontologique ne peut éviter de reposer sur l'unité $d u$ rapport auquel il nous convie. Quelle que soit la vindicte des protagonistes, un "ring" commun reste [carrément]

12. E. Husserl, op. cit. \$ 5, p. 17. Un commentateur de Hegel reprendra à toute fin pratique le mot à mot du même énoncé : "Mais qui a éloigné le monde sensible de la pensée sinon la pensée elle-mème? "E. Fleischmann, La Philosophie politique de Hegel Paris, Plon, 1964, p. 278.

13. "Des intuitions sans concepts sont aveugles, "écrit-il dans la Logique transcendantale de sa Kritik der reinen Vemunft.

14. On citera de mémoire la pertinente remarque du Hongrois Arthur Koestler, l'auteur de Zéro et l'Infini : Il est bien que les spécialistes se spécialisent, l'erreur survient lorsqu'ils gènéralisent. 
incontournable. Un champ de bataille est toujours un champ d'amour - de la bataille, de la victoire ou de la liberté - avant que d'être à l'occasion un champ d'honneur. Et vice versa. Hegel exprime cela dans une puissante formule : l'Identité de l'Identité et de la NonIdentité : Identität der Identität und der Nichtidentität ${ }^{15}$.

Un discours sensé nous appuie rigoureusement au mur de la rationalité. L'interrogation sur l'a-rationalité elle-mēme doit se plier à cette exigence, sans quoi l'examen de la question s'évapore en un flatus voci où tout un chacun a beau jeu d'affirmer posséder le vrai puisqu'il demeure le seul à comprendre le sens de sa propre parole. Or comme nous l'indiquait jadis le poète satirique romain Caìus Lucilius, "savoir n'est pas savoir si personne ne sait ce que l'on sait ${ }^{16}$."

D'aucuns reviendront pourtant à la charge contre de telles conclusions en avançant une suprēme barricade : l'Absolu. La raison, affirme-t-on, ne sait pas tout. La vérité lui échappe parce que l'essentiel ne s'adresse pas à la raison. Peu importe ici que cet Absolu soit nommé Dieu, l'Amour, le Sacré, la Vie, le Beau, le Sens ou l'Un. Ce qu'il faut retenir, c'est le sort privilégié que l'on réserve à un type de connaissance qui considère la raison comme une "faculté " quelque peu anémique, pour ne pas dire indésirable.

Ici ce sera l'irrationalisme romantique lou les philosophies du sentiment) qui, plus particulièrement, fera l'objet des foudres de notre auteur. Bien qu'il ne soit pas toujours aisé de départager en son texte la cible visée, Hegel semble à cet égard regrouper sous un même tenant les "nouveaux "idéalismes allemands: dans la forme esthétique d'un Schiller ou des frères Schlegel (Frédéric surtout), religieuse d'un Jacobi et/ou sentimentale d'un Schleiermacher. Le tableau critique incorporera aussi Schelling, son grand ami d'avant 1807. Mème la poésie d'Hölderlin et l'"idéalisme magique " d'essence fichtéenne de Novalis n'échappent point à la plume hégélienne.

De façon générale, on assisterait chez eux à une réaction systématique au moralisme abstrait de Kant; d'où "cet idéalisme, qui remplacerait le dogmatisme des affirmations [entendez le kantisme], /par un dogmatisme des assurances subjectives lals ein versichernder Dogmatismus) ${ }^{17}$."

15. Entre autres passages, Log. $(J)$, p. 63 (V, 74). On comprendra mieux maintenant ce qu'a voulu signifier l'auteur: "Au fondement de tout notre agir, se trouve /la présupposition de l'unité de la pensée et de l'étre (die Voraussetzung der Einheit des Denkens und des Seins zugrunde). Nous faisons cette présupposition en tant qu'êtres rationnels, /en tant qu'êtres pensants (als denkende Wesen). " Enc.-3, 8 465, add., p. 561 (X, 284). Incidemment, la polysémie de ring sert bien notre propos : l'arène est également une alliance (bague, anneau). Le maitre aurait sans doute apprécié.

16. L'universalité du savoir, dit le Hegel de la Phénoménologie, est " la vérité susceptible d'ètre possédée par toute raison consciente de soi (Wahrheit [ist]. welche fähig ist das Eigentum aller selbstbewußten Vermunft zu sein)". car " toute vérité n'est telle que pour une conscience qui sait (alle Wahrheit ist nur als wissendes Bewußtsein), "lui répond en écho celui de l'Esthétique. Ph., p. 60 (III, 65), et Esthétique, traduction de S. Jankélévitch, Paris, Flammarion, Champs, 1979 [1944], tome I, p. 199 (XIII, 191).

17. Ph.66), p. 129 (128). Bien qu'elles puissent s'apparenter à l'aspect dogmatique de la religion chrétienne, Jacobi insistera toutefois avec force détails pour marquer la distance qui sépare ses thèses de ladite certitude intėrieure. 
Sans doute parce que cette conception de l'Absolu demeure profondēment enracinée dans les esprits, Hegel travaillera ardemment sa vie entière (et bien avant Marx et Nietzsche) à démystifier ce monde-vérité inaccessible à la raison ${ }^{18}$. Comme dans les discussions précédentes, on maintient derechef une dualité. Il y a la réalité - qui cette fois intégrerait la raison - et l'au-delà de la raison et, subséquemment, de la réalité. La conséquence fondamentale de cette approche conduit évidemment à devoir admettre que la raison ne peut discourir de cet Absolu dont elle est exclue. On ne réfléchit pas l'Absolu : on le reçoit, on le sent, on le vit, car il se révèle intérieurement par la belle-âme (die schöne Seele), "cette génialité morale qui sait que la voix intérieure de son savoir immédiat est /voix divine (göttliche Stimme) ${ }^{19}$. "Et puisque la raison se voit contestée au départ, tout commentaire devient du même coup totalement superflu sinon dérisoire.

En définitive, c'est un : "à prendre ou à laisser !" Pascal considère à cette croisée des chemins, advienne que pourra, qu'il faut parier sur Dieu. De toute façon, on ne saurait perdre sur ce coup de dés puisque, bien sùr, l'infini nous attend en promesse. Rousseau va dans le même sens en avançant par la bouche du Vicaire savoyard que l'homme " se décide malgré lui de manière ou d'autre, et il aime mieux se tromper que de ne rien croire" - quitte à opter pour le néant ${ }^{20}$.

Suspendre l'Ultime, le Vrai, Dieu même, le cas échéant de son existence, à une loterie de la sorte apparait aux yeux du penseur allemand comme un contresens. Il y a là une contradiction tout à fait insoutenable. On dépose l'univers du Sens sur le tapis vert du monde comme une vulgaire somme d'argent. En un mot, on assujettit le Vrai à un acte de foi qui, par définition, peut revêtir tous les masques sans qu'aucune réflexion ne soit permise sur les conditions de sa légitimité. En ce sens il y a une vérité pour chaque conscience, qui du reste se replie ou se recroqueville sur sa conviction. Or si la vérité devient histoire de conscience, peut-on persister à conserver le concept mème de vérité ? "Une assurance nue a autant de poids qu'une autre "... Nous sommes dans l'indétermination de la conviction $^{21}$.

Le rapport entre la conscience et ce monde - que l'on considère privilégié dans l'exacte mesure où il est strictement personnel laisse ainsi une porte ouverte à tous les possibles et donc à toutes les erreurs. Nous sombrons, dit Hegel, dans une voie où "tout ce qui est /superstition et service des idoles (Aberglaube und Götzendienst) est

18. Voir la note complémentaire en fin de texte.

19. Ph.-2. p. 186 (III, 481). Cf. Enc.-3, 5471 , rem., p. 269.

20. C'est nous qui soulignons cet extrait de L'Émile. Nietzsche ne tenait pas un autre discours: "L'homme aime mieux vouloir le néant que ne pas vouloir. "La Généalogie de la morale [1887], III, § 28.

21. "Ein trockenes Versichem gilt aber gerade soviel als ein anderes. "Ph, p. 68 (III, 71). La * certitude immédiate de soi-même [...] d'une façon générale est l'arbitraire de l'être singulier et la contingence de son être naturel inconscient (unmittelbare Gewißheit seiner selbst (..) überhaupt die Willkür des Einzelnen und die Zufälligkeit seines bewußtlosen natürlichen Seins) ", ajoute l'auteur en Ph.-2, p. 179 (III, 473). C'est dire qu'elle " met un contenu arbitraire (beliebigen Inhalt) dans son savoir et son vouloir. "Ibid., p. 186 (III, $481)$. 
qualifié de véritét ${ }^{22}$. Si le référent de la vérité de ma certitude réside en ma propre conscience, tout contenu de cette dernière se trouve dès lors marqué du sceau de la "vérité *. D'où la conséquence invraisemblable que le faux égale le vrai : tout ce qui brille devient or.

Ainsi, la thèse qui soutient que la raison ne peut légiférer ni se prononcer sur l'Absolu - qui serait pourtant lui-mème la source dernière de tout sens - chemine vers des implications totalement contradictoires. Il faut se rendre à l'évidence que soustraire l'Absolu à la raison signifie le remettre tout bonnement au premier venu.

Il est de plus très étonnant que l'on conserve tout de mème à la raison ses droits "en dehors" de cet univers tabou. La raison, en effet, peut continuer à philosopher, à manipuler les théorèmes mathématiques et à s'échiner à comprendre le monde. Pourtant le Vrai, le Sens, lui échappe. L'auteur de la Phénoménologie a cerné ce paradoxe dans une formule extraordinairement démystificatrice, où il constate que cette vision du vrai :

présuppose que l'Absolu se trouve d'un côté, et elle présuppose que la connaissance se trouvant d'un autre cóté, pour soi et séparée de l'Absolu, est pourtant quelque chose de réel. En d'autres termes, elle présuppose que la connaissance, laquelle étant en dehors de l'Absolu est certainement aussi en dehors de la vérité, est pourtant encore véridique ${ }^{23}$.

On est définitivement empetré dans un non-vrai vrai et un vrai non vrai. La raison est vraie en son lieu, local, hors duquel elle tomberait dans l'erreur. D'autre part, l'Absolu, qui est le vrai, devient l'illustration du faux en se réduisant au contenu particulier de la conscience individuelle. C'est à ne plus s'y retrouver.

Un dernier rempart semble toutefois subsister sur les vestiges de cette problëmatique de l'Absolu-Vrai. On prétend que la déconstruction hégélienne rate son objectif car il n'y aurait qu'un Absolu, bien que celui-ci puisse demeurer objet de la conscience personnelle. D'où l'inanité apparente du jugement de notre auteur qui, lui, voit plutôt en ce dédale la multitude des "absolus "de toutes les consciences. Fort bien, répond Hegel. Mais alors, comment s'assurer que l'Absolu dont on parle est bien le même pour chacun, et qu'il exprime ce vrai sans lequel toute certitude - de la foi comme de la raison - s'estompe dans l'aléatoire?

À nouveau, deux possibilités s'offrent à nous, poursuit-il. Ou bien je demeure sur une conviction, et je glisse vers les contradictions que l'on vient tout juste d'évoquer; ou bien je travaille à identifier cet Absolu de façon à établir un dialogue avec les autres "croyants", dialogue qui se montre d'une importance cruciale dans le projet d'élucidation du contenu de cet Absolu. Comment savoir, en effet, si réellement il n'y a qu'un seul Absolu - auquel répondraient toutes les consciences - s'il demeure précisément absolument inconnu?

Affirmer que cet Absolu se nomme Dieu, le Vrai ou le Sens ne nous avance guère, une fois de plus. Car si l'on s'en tient à ces termes génériques, on laisse de nouveau à la conscience la liberté de leur assigner le contenu qu'il lui plaira bien de choisir. Par ailleurs, ne pas leur " soutirer " de signification précise nous laisse choir dans

22. Enc, \& 72, p. 337 (VIII, 162). Cf. Enc. § 74 et 77, p. 338 et 341-342.

23. Voir la note complèmentaire en fin de texte. 
la généralité vide. LAbsolu, Dieu ou le Vrai sont des concepts merveilleux dans l'univers de la représentation. Mais quelle est leur signification? Représentent-ils réellement plus qu'un assemblage de lettres si l'on sait que "les mots de divin, d'absolu, d'éternel, etc., n'expriment pas ce qui est contenu en ewx * ? C'est pourquoi il devient primordial, continue le philosophe, de prendre conscience que "le commencement, le principe ou l'Absolu, dans son énonciation initiale et immédiate, est seulement l'universel ${ }^{24}$."

Si ces concepts revètent une signification, il s'avère nécessaire de la révéler. Or, est-il dit, révéler le sens de Dieu ou de l'Absolu c'est le réduire aux catégories de notre raison qui le dénaturent. Dieu est infini et dépasse tout entendement qui chercherait à le dé-finir, et donc à le dé-limiter. Dieu est insaisissable. Soit, rétorque une fois de plus le penseur. Mais alors le problème reste entier. S'il est impossible d'identifier l'Absolu, il apparait du coup qu'on ne saurait affirmer que c'est au mème Absolu que se rëfèrent les consciences qui estiment en faire l'expérience. On revient toujours aux mêmes apories : l'insaisissable est perméable à tous les prédicats et ainsi, inévitablement, à la contradiction intrinsèque.

Le refus de réfléchir l'Absolu autorise la réduction de celui-ci à des abstractions, sinon sa prostitution derrière le rideau des différents discours dont chacun l'affublera. Un Absolu "intouchable " demeure à jamais le résultat d'une fuite - de "la peur de la vérité ", écrira froidement le philosophe. En un mot: un simple refuge $^{25}$. Littëralement, l'inexprimable ne dit rien. C'est ce qui lui permet d'en dire déjà trop. L'auteur rend l'idée par des formules très concises :

L'indicible - sentiment, sensation - n'est pas ce qu'il y a de plus excellent, de plus vrai, mais ce qu'il y a de plus insignifiant, de moins vrai $[\ldots]$ car là où il n'y a aucune déterminité, aucune connaissance non plus n'est possible. La lumière pure est l'obscurité pure $e^{26}$

Ces " cris " de raison parcourent l'œuvre hégélienne de façon systématique depuis la Phénoménologie: "Ce que l'on nomme l'inexprimable n'est pas autre chose que le non-vrai, le non-rationnel, /le seulement visé (das bloß Gemeinte)", aux derniers souffles de

24. "Die Worte des Göttlichen Absoluten, Ewigen usw. das nicht aussprechen, was darin enthalten ist [... Der Anfang, das Prinzip oder das Absolute, wie es zuerst und unmittelbar ausgesprochen wird, ist nur das Algemeine. "Ph, p. 19 (III, 24 et 25 ). Nous soulignons.

25. Voir notre "Hegel, Nietzsche, Freud en miroirs. Penser aimer mourir * Dans la revue Science et Esprit, 1996, vol. 48, n² 2, p. 185-204.

26. "Das Unsagbare, Gefühl Empfindung, ist nicht das Vortrefflichste, Wahrste, sondem das Unbedeutendste, Unwahrste [...] denn wo keine Bestimuntheit ist, da ist auch keine Erkenntnis möglich Das reine Licht ist die reine Finsternis. "Enc., $\$ 20$, rem., p. 287 et $\$ 36$, add., p. 492 (VIII, 74 et 105). Nous soulignons. Il faut entendre ici le mot * in-signifiant " ([Un] bedeutendste) en son sens étymologique (soustrait à la signifiance), et ne pas lui insuffler une valeur moralisatrice ou psychologisante. Hegel écrira également que : * si quelqu'un, à propos de quelque chose, en appelle [...] à son sentiment, la seule attitude à prendre est de le laisser là où il est, car de la sorte /il se refuse à la communauté de la rationalité (er sich dadurch der Gemeinschaft der Vemünftigkeit verweigert, il se retranche dans sa subjectivité isolée, dans la particularité. "Enc.(G), § 447, rem., p. 401 (X, 248). Revoir supra, note 16. 
l'Encyclopédie : "Croire (Glauben) ne signifie rien d'autre que ne pas progresser jusqu'à une représentation déterminée, /ne pas vouloir s'intéresser davantage au contenu (auf den Inhalt sich weiter nicht einlassen wollen $)^{27}$."

Or ce contenu, loin d'être envisagé comme la limite du philosopher, en constitue au contraire le véritable point de départ et ne cessera d'en ètre l'objet ${ }^{28}$. Puisque rien de signifiant ne peut ètre dit hors de la raison, et que celle-ci se dévoile comme l'irréductible source de sens en deçà et au-delà de laquelle la vérité devient mot léger comme neige au vent, il devient clair que l'entreprise philosophique braquera son discours sur la raison elle-mème. Si en effet la philosophie cherche "la vérité des choses", elle cherche littéralement leur raison, le sens qui les habite.

Il s'agira donc de saisir la rationalité du réel en n'oubliant pas un instant - désormais - que là où l'on sera tenté le cas échéant d'oublier, de perdre ou de "dissimuler "la raison, ce mouvement de retranchement ne pourra avoir de sens que s'il s'appréhende, se comprend et se justifie lui-mème. Et devant quel assesseur une fois encore cette attitude pourrait-elle s'interroger, se critiquer, sinon la raison $^{29}$ ?

À partir de ces lignes, s'impose une cruciale constatation: la Raison n'est point un postulat du penser hégélien. La Raison demeure l'horizon sur lequel se dessinent tous les possibles - connus ou à connaitre. Hegel n'a pas "choisi "la rationalité comme d'autres philosophes "optent "pour la vertu, la substance ou la matière. Comme nous tous, il est choisi par elle. Pour en avoir pris conscience et avoir désiré comprendre cette Raison, il récupère déjà pour ainsi dire tout discours de sens. À ce titre, l'auteur demeure indépassable, et nous n'en finirons sans doute jamais de débattre avec et autour de lui.

27. Ph., p. 91 (III, 92), nous soulignons, et Enc.(G). § 573, rem., p. 498 (X, 391). Et en outre : "Nous plaçons Dieu au delà de notre conscience rationnelle, nous nous trouvons du même coup affranchis du souci de connaître sa nature et de reconnaitre la présence de la Raison dans l'histoire. La voie est ainsi libre au jeu des hypothèses arbitraires : La pieuse humilité sait fort bien ce qu'elle gagne à de tels renoncements (Wird Gott jenseits unseres vemünftigen BewuBtseins gestellt, so sind wir davon befreit, sowohl uns um seine Natur zu bekümmern, als Vermunft in der Weltgeschichte zu finden; freie Hypothesen haben dann ihren Spielraum Die fromme Demut weiß wohl, was sie durch ihr Verzichten gewinnt). " Raison, p. 60 (41), nous soulignons. L'inexprimable (das Unaussprechliche), dit-il encore, "n'acquiert de la clarté (Klarheit) que lorsqu'il peut /accéder à la parole (zu Worte zu kommen). "Enc.-3, § 462, add., p. 560 (X, 280). À ce titre, il nous faudra admettre que le senti... ment.

28. Voir la note complémentaire en fin de texte.

29. "Quand l'on parle des bormes de la raison (Schranken der Vernunft), c'est encore pis que de parler de fer en bois (hölzemem Eisen] [...] / La raison ne peut tolérer rien d'autre à côté d'elle et encore moins au-dessus d'elle (Die Vernunft kann nichts Anderes neben sich, noch viel weniger über sich leiden). "Enc.-3, \$ 441, add., p. 540 (X, 233) et Philo., p. 226 (197). Nous nous sommes contenté pour l'instant d'appréhender la raison sous l'angle assez large de foyer de sens et source d'intelligibilité: un prochain article, bientôt publié, en fait son objet spécifique et déterminé. 
Certes, sa préhension de la raison reste celle d'un homme, être de finitude. Comme telle, elle se déploie imprégnée d'ambiguïtés, de maladresses aussi, auxquelles nul n'échappe, mème le plus doué. Cette imperfection, indéracinable, adamesque, ne saurait cependant ètre entrevue qu'au nom de la perfection elle-mème, c'est-à-dire de la vérité, fille de la raison... On comprendra mieux dès lors la profondeur des propos qu'il tint quelques jours à peine avant sa mort à Berlin lorsque, au sujet des catégories de la raison, il affirmait que loin de les posséder, nous étions plutôt " possédés par elles (sie uns im Besitz haben)". "La logique, ajoute-t-il à propos de l'homme, constitue sa nature même (seine eigentümliche Natur selbst) ${ }^{30}$. " Dès les premières lignes de son maitre ouvrage, la mème idée s'impose comme phare sur l'infinitude : "Tout ce qui est humain est humain en ce que, et seulement en ce qu'il est produit au moyen de la pensée ${ }^{31}$."

Cette descente vertigineuse vers la Raison, non moins impressionnante qu'inévitable, ne nous amène pourtant qu'à l'orée du travail à accomplir. Car bien pauvre raison serait celle que l'on découvrirait, tel un oreiller douillet, pour mieux s'y endormir à nouveau comme en un Dieu-refuge qui apaise les angoisses et calme les insomnies ${ }^{32}$. Recherchant le vrai, la Raison ne saurait sommeiller : elle est constamment tendue, pour reprendre l'expression de Malebranche, par son désir de comprendre ${ }^{33}$.

Aussi, il ne suffit plus d'affirmer que le monde est rationnel simplement parce que maintenir l'absence totale ou partielle de la raison est une position intenable. Dans cette étude, nous avons en quelque sorte " récolté " la raison en précisant son rôle - théorique en tout discours de sens. Mais si effectivement la réalité n'est pas étrangère à la rationalité, il nous reste à comprendre de quelle façon la première manifeste la seconde. Car, par le détour synthétique de la confrontation de la rationalité à l'"a-rationalité " (et donc de façon indirecte ou seulement négative), on a découvert en effet que le rationalisme hégélien ne constitue pas un point de départ à justifier, mais bien une "conséquence " à expliciter. Cadeau de Grec !

La Raison gouverne le monde, a-t-on déjà dit. Il s'agirait désormais de savoir positivement comment. De la sorte, nous tenterions avec l'auteur d'identifier la structure et de la réalité et de la

30. $\log .(J$. p. 17 et 12 (V, 25 et 20). "Les déterminations logiques (logischen Bestimmungen) en général peuvent être regardées comme des définitions de l'Absolu, comme des défunitions métaphysiques de Dieu (metaphysischen Definitionen Gottes). "Enc., \& 85, p. 348 (VIII, 181).

31. "Ist alles Menschliche dadurch und allein dadurch menschlich daß es durch das Denken bewirkt wird. "Enc., § 2, p. 164 (VIII, 42). "Il n'est pas possible de renoncer à la pensée (Das Denken einmal können wir nirgend unterlassen), " dit-il encore dans Raison, p. 43 (25). De même, "c'est une grande obstination (eir Großer Eingensinn), l'obstination qui fait honneur à l'homme, de ne rien vouloir reconnaître dans sa conviction qui n'ait été /justifié par la pensée (durch den Gedanken gerechtfertigt ist). "Droit, p. 58 (VII, 27). En un mot, penser est indis-pensable.

32. Thème cher à Nietzsche notamment, qui redit combien nous savons " préserver notre ignorance pour jouir, à un degré presque inconcevable. de la liberté, de l'insouciance, de l'imprudence, de la vivacité, de la joie de la vie. "Par-delà bien et mal [1886] \& 24 . Nous soulignons. Traquer la raison pour en faire ultimo l'objet d'une religion serait, en effet, le comble de l'absurde : la traque précédant le troc.

33. Voir la note complementaire en fin de texte. 
raison et, par là, de retrouver l'essentiel des matériaux qui nourrissent le chemin du sens et de la vérité. Comme le professait $\mathrm{Hegel}$, parler de la raison demeure au fond vaine entreprise, car :

Die Vernunft in ihrer Bestimmung gefaßt,

dies ist erst die Sache S $^{34}$

\section{Notes complémentaires}

2. Husserl a toujours maintenu ne pas (vouloir) lire Hegel, comme pour ne pas " contaminer "l'originalité de sa propre réflexion. Ces deux penseurs allemands demeurent, on le sait, les dignes sommités du rationalisme philosophique, d'où peut-être l'intuition du puiné $(\dagger 1938)$ de se préserver du grand frère (Freud adopta semblable attitude face à Nietzsche). Notons au passage que nos philosophes étaient frappés d'une mème horripilation devant tout formalisme. On n'aura qu'à retenir à cet égard les catégories de Lebenswelt et d'intentionnalité, fondamentales chez Husserl, par lesquelles il tenta inlassablement de paver la voie de l'homme donateur de sens - jusqu'à la raison. Et réciproquement. Pour lui comme pour Hegel, le rationalisme cohérent (truisme de bon aloi) est l'antithèse de la rationalisation. Les deux " $\mathrm{H}$ " sont d'ailleurs les co-fondateurs, chacun à sa façon et à un siècle d'intervalle, du grand courant de la Phénoménologie-lequel devait baigner tout notre siècle, jusqu'à la "Déconstruction " du Sujet laborieusement érigé.

L'affrontement cogitatif Husserl-Hegel reste en grande partie à l'origine de ce que la philosophie contemporaine, continentale européenne, produisit de mieux construit avec Heidegger, Merleau-Ponty, Sartre, Ricoeur, pour ne nommer que ceux-là. Nietzsche et Marx, Kierkegaard aussi, se joindront bientôt à la conversation et aux grands débats par filles et fils spirituels interposés. La Phénoménologie se fera de plus en plus psychologique d'une part, politique d'autre part, en concomitance ou en diachronie. Elle deviendra enfin Existentialisme pour la génération d'après-guerre, avant son éclatement (final ?) et pour ainsi dire sa dilution.

18. Par opposition à l'idée largement répandue, nous irions même jusquáa affirmer que ce combat contre l'abstrait atteint son objectif plus radicalement encore chez Hegel que chez ces "Maitres du soupçon ". Modifier le "contenu " de la divinité-en-soi ne nous en libère pas pour autant : tuer ou nier Dieu pour le remplacer par le Prolétariat (ou l'Histoire, sinon le Parti: "Nos athées sont de pieuses gens ", ironisait Max Stirner), par le Surhomme ou, pour continuer dans la lignée du soupçon, par l'Inconscient du psychanalyste ("Dieu est inconscient ", dit le Lacan du Séminaire $x]$, nous enferme en un Absolu nouvelle mode qui se présente comme le "vëritable lieu "d'où le reste tire son sens comme à sa source. On demeure ainsi dans l'abstraction d'un dualisme entre le "Vrai * et ce qu'il conditionne-c'est-à-dire, en langage

34. "Saisir la raison dans sa détermination, voilà la chose. "Raison, p. 69 (49). Voir la note en fin de texte. 
hégélien, dans un Ab-solu d'entendement. Ce qui, sur le plan formel, nous ramène exactement à la foi du charbonnier, sinon à l'image du blanc barbu de notre petite enfance, cousue du gros fil mauve de la catholicité des "Monseigneurs ". "Il ne résulte pas encore d'une pensée, d'une conviction personnelle, écrit le philosophe avec grande finesse, que l'on soit affranchi de l'autorité [...] car notre propre révélation intérieure est une sorte d'autorité (Eigenes Denken, eigene Überzeugung macht es also noch nicht, daß man frei von Autorität sei $[. .$.$] denn daß es aus$ unserer eigenen inneren Offenbarung komme, ist eine Weise der Autorität). "Philo., p. 224 et $223(196+195)$. Nous soulignons. Or, chez Hegel l'Absolu cesse précisément de s'affirmer comme ce lieu - ce tróne : que celui-ci se trouve au fond du coeur, aux il-limites de l'infini ou dans les moyens de production - d'où se révélerait le sens. Il se manifeste au contraire en tout ce qui est et par ce qui est. C'est pour ainsi dire son travail. Et celui de Hegel de le demontrer.

Si par ailleurs, et inversement, l'on prétendait que l'Absolu n'est que le simple résultat, par exemple, de l'absolutisation de la raison humaine, et donc une pure fantasia de l'esprit, on reviendrait tout de même alors à une compréhension unifiée et autonome de la raison. En l'occasion, l'Absolu hégélien n'aurait certes pas un statut identique chez nos critiques - avant la lettre chez un Diderot notamment, digne représentant des Lumières crues françaises, ou le positivisme fin $\mathrm{XIX}^{\mathrm{c}}$, en passant par la réflexion effectivement décapante des Marx et des Nietzsche -. mais tout ce gratin indigné des bondieuseries en vigueur scellerait le pacte hégélien que voici : "Il n'y a qu'une raison, il n'en est pas une seconde, surhumaine; elle est le divin dans l'homme (Die Vernunft ist nur eine; es gibt keine zweite, übermenschliche Vernunft. Sie ist das Göttliche im Menschen)." Philo., p. 146 (123). Nous soulignons.

Quelque absolu qu'il soit l'" Idéalisme " de Hegel, comme nous l'apprend le passage d'allure condillacienne qui suit, reste à cet égard plus près du matérialisme manière de La Mettrie ou de d'Holbach que de l'exaltation ou de l'illuminisme: "Dans la sensation, est présente la raison tout entière, - le matériau intégral de l'esprit (In der Empfindung ist die ganze Vernunft, der gesamte Stoff des Geistes vorhanden). "Enc.-3, § 447, add., p. $546(\mathrm{X}, 248)$. Ce qui cependant ne retient nullement son concepteur d'accorder une très large place à Boehme, et admirative encore, dans son Histoire de la Philosophie. Les grandes pensées, on ne le dira jamais assez, ne se réduisent pas à deux ou trois entourloupettes de manuels didactiques, fort peu pédagogiques le plus souvent. Par définition. Parce que la pensée c'est vivant - comme le lombric dans un sol humide, comme eine schöne Seele follement éprise de sa bien aimée.

23. L'importance du commentaire nécessite la version originale complète : "Sie setzt daß das Absolute auf einer Seite stehe und das Erkennen auf der andern Seite für sich und getrennt von dem Absoluten doch etwas Reelles [sei], oder hiermit, daß das Erkennen, welches, indem es außer dem Absoluten, wohl auch 
außer der Wahrheit ist, doch wahrhaft sei. *Ph., p. 66-67 (III, 70). Hegel ne manquera pas, après 1807 , de revenir régulièrement sur cette universelle et persistante contradiction. Depuis l'introduction de sa Logique en 1812 : * Résultat étrange, étant donné que l'infini est le rationnel, que de dire : la raison n'est pas capable de connaitre le rationnel (Ein sonderbares Resultat, indem das Unendliche das Vernünftige ist, zu sagen, die Vernunft sei nicht fähig, das Vernünftige zu erkennen) ", jusqu'à l'avant-propos de la $3^{\mathrm{e}}$ édition de L'Encyclopédie, en 1830 : "La religiosité, tout ensemble celle qui est pieuse et celle qui réfléchit, /en est venue à trouver la satisfaction la plus haute là où il n'y a pas de contenu (dazu gekommen ist, die höchste Befriedigung ohne Inhalt $\mathrm{zu}$ finden) ", en retenant au passage l'allocution $\mathrm{de}$ 1818: "On ne connaitrait rien de vrai ( $d a \beta$ nichts Wahres erkannt), mais que seul ce qui est sans vérité (Unwahres), temporel et passager jouirait en quelque sorte de l'avantage d'être connu. "Ce qui en outre implique, nous dit le "Concept général " de la Logique, achevé à l'automne de 1831: "L'absurdité qu'il ne serait pas nécessaire de connaître un objet tel qu'il est en soi pour en avoir une vraie connaissance (So ungereimt ist eine wahre Erkenntnis, die den Gegenstand nicht erkennte, wie er an sich ist). " Respectivement: Log.-1, p. 28 (V, 52), Enc., p. 143 \& 147 (VIII, $37+X, 402$ ), et $\log .(J)$, p. 31 (V, 39). Nous ne résistons pas à l'impulsion de revenir soudain à Platon, quelque 2400 ans en arrière, ne fút-ce que pour mesurer à l'aune de nos piétinements la lenteur du concept dans l'histoire des hommes (bien qu'à certains égards, l'élève de Socrate soit notre exact contemporain : l'univers a tout de même exigé 15 milliards d'années, en effet, pour nous offrir la poiêsis d'un être capable de poésie filiation étymologique riche d'enseignement, et au surplus implacable désaveu du poète... qui ne "fait "rien). C'est le Sophiste (248e - 249a, traduction Chambry) qui retentit comme en écho de désespérance du fond des siècles :

Mais par Zeus I nous laisserons-nous si aisément persuader que le mouvement, la vie, l'äme, la pensée n'ont vraiment pas de place en l'ètre absolu, qu'il ne vit ni ne pense, et que, vénérable et sacré, dénué d'intelligence, il reste figé et sans mouvement?

Éternels orphelins de sens que nous sommes, par indigence de totalisme. Par Kronos ! qu'il est loin le cœur de la tendresse des choses.

28. "La raison aborde le monde avec la croyance absolue de pouvoir poser l'identité et élever sa certitude à la vérité, et avec l'impulsion à poser aussi comme du néant l'opposition qui est pour elle en soi du néant. Ce processus est d'une façon générale la connaissance (Die Vernunft kommt an die Welt mit dem absoluten Glauben, die Identität setzen und ihre Gewißheit zur Wahrheit erheben zu können, und mit dem Triebe, den für sie an sich nichtigen Gegensatz auch als nichtig zu setzen. Dieser Prozeß ist im allgemeinen das Erkennen). "Enc. \$ 224 et 225, p. 454 (VIII, 378). Ce qui est divisé ou séparé, ajoute le professeur dans une formule bien à lui, "la philosophie l'unifie, unissant ainsi le 
dimanche de la vie à la journée ouvrable ldas Faßt die Philosophie in Eins zusammen und vereint so den Sonntag und Werktag des Lebens). " Philo., p. 250 (220).

Il est fait remarquer que l'on commet souvent "l'inversion consistant en ce que ce qui est nommé rationnel est ce qui relève de l'entendement, /alors que ce qui est nommé irrationnel est bien plutôt un commencement et une trace de rationalité (aber irrational, vielmehr ein Beginn und Spur der Vernünftigkeit ist). " Enc., \$ 231, rem., p. 458 (VII, 384), nous soulignons. On constate à nouveau que la soi-disant "impitoyable " raison chez Hegel (impie non moins que pitoyable, diront d'aucuns) ne constitue pas une Néron de la personnalité humaine, fût-ce la sienne propre. Si réellement rien n'échappe au rationnel, on ne saurait conclure de là, en effet, que "tout le reste "- disons : la nonraison - est bavardage ou catinage. C'est exactement l'inverse qui se produit. Ailleurs, le philosophe avance que "le moment vrai et spéculatif est celui où l'entendement se refuse à entrer, lui qui précisément appelle le concept l'inconcevable [car...] L'entendement ne trouve dans la spéculation que des contradictions, il en reste à la distinction et ne peut comprendre le concret (das Wahre und Spekulative [Moment] ist es, in welches einzugehen sich der Verstand weigert, der immer gerade den Begriff das Unbegreifliche nennt [...] Der Verstand findet überall in dem Spekulativen nur Widersprüche; er bleibt beim Unterschied stehen, kann das Konkrete nicht fassen). "Droit, § 7, rem., p. 76 (VII, 55) et Philo., p. 239 (210). Nous soulignons. Pour l'entendement, la raison est aussi insaisissable qu'une émotion. Ou la folie.

Aussi, lorsque Hegel pourfend le "sentiment " avec la dernière énergie, comme nous venons de l'entrevoir, ce n'est pas dans l'espoir de l'annihiler ou de s'en libèrer comme d'une tare. Si on sait déjà que "le réel est rationnel " [Droit, p. 55 (VII, 24)], qu'il n'y a "qu'une raison en l'homme " [Philo., p. 146 (123)] et qu'elle " gouverne le monde "[Raison, p. 47 (28)], en revanche il ne fut jamais affirmé que cette rationalité était ou devait être mandatée de quelque façon pour dissoudre ce réel. Il s'agit bien plutôt de comprendre que raison, sentiment et monde sont saisis d'une méme rationalité. C'est la séparation, la dichotomie qui, pour lui, est indéfendable parce que littéralement injustifiable. Hegel parlera de " la vérité du cœur et du vouloir et, ce qui revient au mème, leur rationalité effective (die Wahrheit und, was dasselbe ist, die wirkliche Vernüftigkeit des Herzens und Willens). "Au même paragraphe, il ajoute : Le rationnel, qui se trouve dans la structure de la rationalité comme quelque chose de pensé, /est le même contenu (ist derselbe Inhalt) que celui du bon sentiment pratique, mais (aber) dans son universalité et sa nécessité, /dans son objectivité et sa vérité (in seiner Objektivität und Wahrheit). "Enc.(G), \& 471, rem., p. 420 (X, 291). Nous soulignons.

La raison ne survole donc pas "ce qui est ": elle l'embrasse et le com-prend dans toute l'extension de ces termes. Ce qui lui est d'ailleurs en quelque sorte une activité congénitale (par distinction d'un acte second), comme l'a magistralement démontré 
la Phénoménologie. La " conscience insignifiante du ceci" , prégnante et pour ainsi dire "absolue " en début d'humanité (au sens animalien : présence entière à l'immédiatetē) - sur le plan ontogénique aussi bien que phylogénique - se révèle d'emblée happée, dès l'abord, par le ressort de sa propre raison. La conscience est entrainée par-delà elle-même, ne sachant pas encore qu'elle est tirée vers l'avant par elle-mēme - et moins encore qu'en bout de route elle rencontrera un grand Autre qui s'appelle Moi. En termes concis: "Un tel chemin vers la science est lui-même déjà science (Ist dieser Weg zur Wissenschaft selbst schon Wissenschaft). "Ph., p. 77 (III, 80).

Hegel l'a fort bien exprimé dès le début de l'ouvrage, en préface: "L'intelligible est ce quỉ est déjà connu, l'élément commun de la science et de la conscience non scientifique, laquelle peut ainsi s'ouvrir immédiatement un passage vers la science (Das Verständige ist das schon Bekannte und das Gemeinschaftliche der Wissenschaft und des unwissenschaftlichen BewuBtseins, wodurch dieses unmittelbar in jene einzutreten vermag). " Ph. p. 14 (III, 20)/Ph.(66), p. 37 (36). Traduction légèrement retouchée, et soulignèe. En un mot: la conscience pré-scientifique est par soi pré-scientifique. La raison peut ètre enfouie, inconsciente, répugnante, ridicule, affolée, paresseuse ou violente, que sais-je encore? Mais elle n'est jamais "à cōté ". Toujours dedans fût-ce derrière, comme... une arrière-pensée littéralement: "Die Logik hinter dem BewuBtsein," ou "hinter seinem Rücken" (véritable symétrique - ou anté-calque ? - au rapport Es /Ich freudien). Ph., p. 77 (III, 80) et/ou Enc. § 25, rem., p. 290 (VIII, 92).

L'auteur ne rate jamais une occasion de tancer "l'esprit de sérieux ". La raison, loin d'être un dessiccatif de la vie, en est le grenouillement même. C'est toute la nuance entre la Raison (Vernunft) et son second, certes indispensable mais insuffisant, à savoir l'Entendement (Verstand) - qui est à la première, dans le langage de Conan Doyle, ce.que Watson est à Holmes : c'est bien Sherlock qui cogite, fait les spéculations et résout, ultimement, les problèmes.

La raison hégélienne est partout non pas parce qu'elle aurait tout détruit sur son passage, mais parce qu'elle imprègne tout. Elle n'est pas une Guerrière, raffolant de sang et de souffrance, mais une Amoureuse. Quoique attention! pas une nymphette alanguie de cinéma, prélassée dans les draps de soie naturelle, vie-sionnant distraitement l'existence paradant devant elle. Elle s'apparente plutōt - ce qui au demeurant n'est pas sans rappeler l'Athéna des Grecs - à la dame qui tient les couleurs dans la si belle et si puissante Liberté guidant le peuple, d'Eugène Delacroix (toile qu'il peignit en commémoration des "Trois Glorieuses " de la monarchie dite de Juillet, et incidemment à la même période où Hegel écrivait, ou peaufinait, en 1830 , nombre des textes cités prêcédemment).

Déesse Raison n'a cure des draps et des miroirs. Elle a trop à faire avec l'embellie du monde pour se préoccuper de prétendants transis à ses pieds devant sa superbe, sa fougue et sa rigueur. C'est peut-être pour cela d'ailleurs qu'Homère fit de la 
jeune femme casquée une vierge farouche, tout fin prête pour le combat dès sa naissance du cerveau de son père. Et par Éros ! d'autant plus désirable. Jeanne d'Arc avant Jeanne d'Arc.

33. Le Féminin nous aspire vers le haut, a écrit Goethe. Hegel dit la mème chose autrement de la raison-dūt-on en passer par l'abysse. Il nous semble voir l'un se laisser guider par l'Adagio d'Albinoni, l'autre par la poésie de Schiller en finale de la Neuvième Symphonie de Beethoven, au reste composée à l'époque où le penseur était lui-mème au sommet de son art lon se souviendra rétrospectivement que le philosophe a également terminé le "Savoir absolu" sur les vers du mèmel. Si on nous autorise à poursuivre la digression on apprendra que les deux compatriotes, et ponctuels frères temporels (qui, sauf erreur, ne se connaissaient que de réputation), composèrent leur premier grand ouvrage respectif, soit la $5^{e}$ Symphonie et la Phénoménologie, exactement au mème moment (pendant ce temps à Weimar, Goethe achevait le premier Faust!). Nous étions en 1806, alors que Napoléon brillait au firmament étoilé de "l'Esprit de la Révolution ", naguère saluée avec une émotion presque religieuse par nos... artistes. Pour l'anecdote, et comme par surcroit, on remarquera que les deux surdoués soulignent des traits, et physiques et psychologiques, à certains égards étonnants de similarité. Devant ce faste culturel et politique mêlé de génie dans une atmosphère d'exaltation, nos sixties perdent quelque peu de leur séduction (et ne parlons pas de l'actuellement-actuel - ce serait trop cruel - où la priorité semble résider dans l'exploit de faire tourner le plus rapidement possible un pot de moutarde trois fois autour de la planète, at the best price possible, in English too please and only. Bref le laminage de tous à la faveur de l'ouverture! " for the same. Quelque chose comme la loi de la jungle en complet veston). Terminons par une note agréable, une suggestion : la lecture et l'écoute simultanée des deux œuvres citées. Nous ne saurions trop déconseiller pareil amalgame, cependant, à la lecture des travaux pointus de maturité. S'il est vrai que $M$. Ludwig est sans doute, avec Wagner, le plus philosophe des compositeurs et Georg Wilhelm le plus konkret des penseurs, la Logik et la Symphonie en do mineur tentant de s'approprier conjointement un même cerveau feraient à nos yeux figure de hölzern Eisen, pour reprendre le mot déjà rapportè à la note 29 .

34. S'inspirant de l'Apocalypse (III, 16), Hegel insiste avec vigueur sur le fait que "la raison ne se contente pas de l'approximation, car celle-ci n'est ni chaude ni froide et doit être vomie (die Vernunft sicht nicht mit der Annäherung, als welche weder kalt noch warm ist und darum ausgespien wird). "Droit, p. 58 (VII, 27). "La tâche de la philosophie est d'appréhender rationnellement la raison ", dit par ailleurs excellemment $M$. Bourgeois dans un commentaire de sa traduction de l'Enc., note 8, p. 285. Pour le dire d'un seul élan: "La Science a seulement pour tāche d'amener à la conscience ce travail propre de la raison de la Chose (Die Wissenschaft hat nur das Geschäft, diese eigene Arbeit der Vernunft der Sache zum Bewußtsein zu bringen). "Droit, \$ 31 , 
rem., p. 90 (VII, 85). C'est le programme annoncè de notre réflexion à paraitre en 1997, et intitulè : " La Logophonie dialectique : Sujet - Négativité - Résultat - Réconciliation. Pour introduire à la Raison hégélienne. "Plus largement, nous renvoyons à notre ouvrage, Hegel ou de la Raison intégrale, à paraître bientôt à Paris et à Montréal aux Éditions L'Harmattan.

Chercheur indépendant

Lac Beauport 


\section{Cfurres de Hegel}

Tous les textes cités, sauf items 3,5 et 7 , renvoient à l'édition suivante : WERKE IN ZWANZG BÄNDEN. Frankfurt am Main, Suhrkamp Verlag, 1969-1971. La pagination de l'édition allemande (entre parenthèses et précédée du tome le cas échéant) suit celle de la traduction.

1. Enc. / Enc.-3 Encyclopédie des sciences philosophiques. I, La Science de la Logique, et III, La Philosophie de l'Esprit [1817, 1827, 1830]. Traduction, présentation et annotations de B. Bourgeois. Paris, Vrin, 1979 et 1988.

2. Enc.(G)

Encyclopédie des sciences philosophiques en abrégé [1830]. Traduction de M. de Gandillac. Paris, Gallimard, NRF, 1970.

3. Philo. \& Philo.-2 Leçons sur l'histoire de la Philosophie. Introduction: Système et histoire de la Philosophie [1816, 1820-1830]. Traduction de J. Gibelin. Paris, Gallimard, NRF, 1954, 2 tomes. Traduction de : GESCHICHTE DER PHILOSOPHIE (EINLEITUNG). Texte établi par J. Hoffmeister. Hambourg, Felix Meiner Verlag, 1959.

4. Ph. / Ph-2 La Phénoménologie de l'Esprit [1807]. Traduction et notes de J. Hyppolite. Paris, AubierMontaigne, 2 tomes, 1939 et 1941.

5. Ph. (66)

La Phénoménologie de l'Esprit [1807]: "Préface". Nouvelle traduction, introduction et notes de J. Hyppolite. Paris, Aubier, la Philosophie en poche, Édition bilingue, 1966 [Réimpr : 1978].

6. Droit Principes de la Philosophie du Droit ou Droit naturel et science de l'État en abrégé [1821]. Traduction, présentation et commentaires de $R$ Derathé, en collaboration avec J.-P. Frick. Paris, Vrin, 1982.

7. Raison La Raison dans l'Histoire. Introduction à la Philosophie de l'Histoire [1822-1830]. Traduction, introduction et notes de $K$. Papaioannou. Paris, U.G.E., 1965. Traduction de : DIE VERNUNFT IN DER GESCHICHTE. Texte établi par J. Hoffmeister. Hambourg, Felix Meiner Verlag, $5^{\mathrm{e}}$ édition, 1955.

8. Log.-1, 2, 3 Science de la Logique. I, L'Être [1812]; II, L'Essence [1813]; III, Le Concept [1816]. Traduction, présentation et notes de G. Jarczyk et P.-J. Labarrière. Paris, Aubier-Montaigne, 1972, 1976 et 1981.

9. Log.(J) Science de la Logique. I, L'Être [1831]. Traduction de S. Jankélévitch. Paris, AubierMontaigne, Bibliothèque philosophique, 1972 [1947]. 